\title{
Association of Matrix Metalloproteinases and Their Tissue Inhibitor With Disease Activity and Development in Spondyloarthropathy and Inflammatory Bowel Disease
}

\author{
Dursun ELMAS, ${ }^{1}$ Ali ŞAHİN, ${ }^{2}$ Orhan KÜÇÜKŞAHİN, ${ }^{3}$ Nuran TÜRKÇAPAR, ${ }^{4}$ Murat TÖRÜNER, ${ }^{5}$ \\ Hülya ÇETİNKAYA, ${ }^{5}$ Emre KÜLAHÇIOĞLU, ${ }^{1}$ Alexis K. OKOH, ${ }^{1}$ Murat TURGAY ${ }^{4}$ \\ ${ }^{1}$ Department of Internal Medicine, Medical Faculty of Ankara University, Ankara, Turkey \\ ${ }^{2}$ Department of Internal Medicine, Division of Rheumatology, Medical Faculty of Cumhuriyet University, Sivas, Turkey \\ ${ }^{3}$ Department of Internal Medicine, Division of Rheumatology, Medical Faculty of Yildırım Beyazit University, Ankara, Turkey \\ ${ }^{4}$ Department of Internal Medicine, Division of Rheumatology, Medical Faculty of Ankara University, Ankara, Turkey \\ ${ }^{5}$ Department of Internal Medicine, Division of Gastroenterology, Medical Faculty of Ankara University, Ankara, Turkey
}

\begin{abstract}
Objectives: This study aims to investigate whether clinical measures of disease activity and function in ankylosing spondylitis (AS) and inflammatory bowel disease (IBD) are associated with matrix metalloproteinase-3 (MMP-3), matrix metalloproteinase-9 (MMP-9) and tissue inhibitor of matrix metalloproteinase -1 (TIMP-1), and if MMPs can be more useful than C-reactive protein and erythrocyte sedimentation rate in predicting disease activity in AS.

Patients and methods: MMP-3, MMP-9 and TIMP-1 levels were measured by ELISA in 20 patients with AS, 20 patients with IBD, 20 patients with IBD and AS (35 males, 25 females; mean age 38.1 years; range 19 to 62 years), and 20 healthy volunteers (10 males, 10 females; median age 38.5 years; range 24 to 63 years) as a control group. Bath Ankylosing Spondylitis Disease Activity Index, Truelove-Witts activity criteria for ulcerative colitis, and Crohn's Disease of Activity Index scoring systems were used.

Results: Highest MMP-3 level was in IBD group $(33.51 \pm 59.56 \mathrm{ng} / \mathrm{mL}, \mathrm{p}<0.045)$. MMP-3 levels were significantly higher in patients with IBD and $\mathrm{IBD}+\mathrm{AS}$ than in patients with $\mathrm{AS}(\mathrm{p}<0.007$ and $\mathrm{p}<0.035$, respectively). Highest MMP-9 levels were in the control group $(10.35 \pm 2.61 \mathrm{ng} / \mathrm{mL}, \mathrm{p}<0.48)$. MMP-9 levels were higher in AS group patients than those in IBD and IBD+AS groups, but the difference was not statistically significant ( $<<0.494$ and $\mathrm{p}<0.260$, respectively). Highest TIMP-1 levels were in the IBD group $(8.11 \mathrm{ng} / \mathrm{mL}, \mathrm{p}<0.006)$. TIMP-1 levels of IBD group were significantly higher than both AS and IBD+AS groups ( $p<0.033$ and $p<0.008$, respectively). A statistically significant correlation was detected between serum MMP-3 levels and disease activity and Bath Ankylosing Spondylitis Disease Activity Index score in patients with AS ( $r=0.841, p<0.05)$.

Conclusion: We concluded that serum MMP-3 levels may be a better biomarker than C-reactive protein and erythrocyte sedimentation rate in showing disease activity in AS.

Keywords: Ankylosing spondylitis; inflammatory bowel disease; metalloproteinase-3; metalloproteinase-9; tissue matrix metalloproteinase inhibitor-1.
\end{abstract}

Extra-intestinal involvement is a common manifestation in Inflammatory Bowel Disease (IBD). Joints are the most common site of involvement and the co-existence of IBD with spondylitis or peripheral arthritis (Asymmetric arthritis on lower extremity joints) is considered as Spondiloarthropathy (SpA), "enteropathic arthritis" or "arthritis related to IBD" according to the "European Spondiloartropathy Study Group" criteria. ${ }^{1,2}$ The pathogenesis of extra-intestinal findings in IBD is poorly understood. Current theories suggest the impairment of mucosal immunoregulatory mechanisms in IBD, resulting in the transport of luminal content antigens to the systemic circulation. Also, lack of intestinal mucosal barrier protection mechanism, mucosal secretion of immunoglobulin $\mathrm{A}(\mathrm{Ig} \mathrm{A})$ and defense mechanism of mucosa against foreign proteins

Received: August 23, 2014 Accepted: October 27, 2014 Published online: December 11, 2014

Correspondence: Ali Şahin, M.D. Cumhuriyet Üniversitesi Tıp Fakültesi İç Hastalıkları Anabilim Dalı, Romatoloji Bilim Dalı, 58140 Sivas, Turkey.

Tel: +90 346 - 2580949 e-mail: dralsahin@hotmail.com

@2015 Turkish League Against Rheumatism. All rights reserved. 
in IBD contribute to dysfunction of immune regulation. ${ }^{2}$

Secondary to this predisposing condition, the transport of cytokines, endothelial adhesion molecules, fibrinogen, reactive oxygen metabolites and many other antigens that may arise as a result of intestinal inflammation into the systemic circulation contribute to the extra-intestinal findings in distant organs. ${ }^{3}$ Moreover, intestinal trauma leading to disorders on cell surface and intercellular permeability can result in patients developing inappropriate inflammatory responses to self proteins and environmental factors.

Recent studies have reported the impact of matrix metalloproteinases (MMPs) and their tissue inhibitors on the regulation of intestinal barrier protection mechanism as well as new ulcer formation. These proteins have been shown to be associated with disease activity as well as extraintestinal finding development. ${ }^{4}$

The function of MMPs, a family of $\mathrm{Zn}^{2+}$-dependent endopeptidases, in the degradation of extracellular matrix components during tumor invasion and metastasis as well as their role in the processes of tissue destruction and remodeling during inflammation have been proposed. ${ }^{5-7}$ The natural inhibitors of MMPs are called tissue inhibitors of metalloproteinases (TIMPs). These proteins block enzyme activity by binding to metalloproteinases with great affinity. Their effects on MMPs during tumor progression are twofold; beneficial thus inhibiting MMPs and impairing angiogenesis and harmful by facilitating cancer cell generation and growth. ${ }^{8-10}$

Inflammation of the mucosa in IBD patients has been linked with TIMP expression, correlating inversely with the production of proinflammatory cytokines and the grade of inflammation. ${ }^{11,12}$ The sensitive balance between MMPs and TIMPs defines many physiological processes of the gut as well as severity of articular cartilage damage. . $^{8,9,13}$

The effects of MMPs and their tissue inhibitors on disease activity, erosion, and tissue reconstruction in ankylosing spondylitis (AS) has been previously reported. ${ }^{13}$ A study which investigated the association between serum levels of MMP-3, macrophage colony stimulating factors, and disease activity showed that these molecules are more useful than $\mathrm{C}$-reactive proteins (CRP) and erythrocyte sedimentation rate (ESR) in predicting disease activity in AS. ${ }^{14}$

To the best of our knowledge, the association of MMPs and TIMP-1 with disease activity and development has rarely been investigated in the same experimental setting in SpAs and IBD. In this study, we aimed to investigate whether clinical measures of disease activity and function in AS and IBD are associated with MMP-3, MMP-9 and TIMP-1, and if MMPs can be more useful than CRP and ESR in predicting disease activity in AS.

\section{PATIENTS AND METHODS}

Patients who visited the Out-patient Department Clinics of the Ankara University School of Medicine, Department of Internal medicine, Division of Rheumatology and Gastroenterology between June 2011 and July 2012 were enrolled. The study group consisted of a total of 60 subjects (35 males, 25 females; mean age 38.1 years; range 19 to 62 years) including 20 Turkish AS patients who fulfilled the 1984 modified New York criteria, ${ }^{14} 20$ IBD patients, 20 IBD and AS patients, and 20 age-matched Turkish healthy volunteers (10 males 10 females; median age 38.5 years; range 24 to 63 years) as a control group. The European spondyloarthropathy study group criteria ${ }^{1}$ were used for the diagnosis of spondiloarthropathy. Before the study, written informed consents were obtained from all participants. The study protocol was approved by the local ethics committee and was in accordance with the 2008 Helsinki Declaration.

Age, gender, age of onset of disease, disease involvement, complications (if any), disease activity, treatment protocols and history of therapies with dates were recorded after each appointment. Disease localization and severity were noted according to recent endoscopy reports in patients' files.

We evaluated disease activity in AS patients by using the Bath Ankylosing Spondylitis Disease Activity Index (BASDAI). The Truelove and Witts criteria were used for evaluation of disease activity in ulcerative colitis (UC) patients, and the Crohn's Disease of Activity Index (CDAI) for patients with Crohn's disease. ${ }^{15-19}$ BASDAI scores were calculated independently from treatment in AS 
and AS+IBD groups to evaluate the association between MMP-3, MMP-9, TIMP-1, ESR, CRP, and BASDAI scores.

Eight milliliter of blood obtained from antecubital veins of all patients were allowed to clot in heparin free tubes for 30 minutes, and were then centrifuged at $3000 \mathrm{rev} / \mathrm{min}$ for 15 minutes. The sera were frozen at $-80{ }^{\circ} \mathrm{C}$ immediately after sample collection. Serum concentrations of MMP-3, MMP-9 and TIMP-1 were measured with a quantitative sandwich ELISA (Biovendor Human MMP-3 and eBioscience Human MMP-9, TIMP-1) tool kit according to the manufacturer's instructions. Microplates were read on the EL 312 Microplate ELISA reader at $450 \mathrm{~nm}$ spectrophotometrically. ESR levels were measured automatically by Kimased Auto 60-Vacutest Kima system (Via dell'Industria, Arzergrande (PD), Italy), and CRP levels were measured using the BN II nephelometer (Dade Behring, Siemens Healthcare Diagnostics Inc., USA).

\section{Statistical analysis}

Statistical analyses were carried out using the SPSS statistical package for windows, version 15 (SPSSInc., Chicago, IL, USA). Categorical variables were expressed as percentages and frequencies, continuous variables as median (minimummaximum). Normality of the data was tested by using the Shapiro-Wilks test. Since most of the data were not in normal distribution, nonparametric methods were used for data analysis.

For MMP-3, MMP-9, TIMP-1, ESR, CRP, age and disease duration, the Kruskal Wallis test was used to analyze group differences. Correlations between variables were shown using the Spearman's rank correlation test. Differences among groups were analyzed with non-parametric post-hoc double group tests.
Correlations between BASDAI scores and MMP-3, MMP-9, TIMP-1, ESR, and CRP were demonstrated with the Spearman's rank correlation test. Correlation between BASDAI scores and disease severity was analyzed using the Mann-Whitney $\mathrm{U}$ test. Receiver operating characteristic analysis was used to compare the whole spectrum of sensitivity and specificity of different markers. Statistical significance was accepted at a $p$ value of $<0.05$.

\section{RESULTS}

The mean duration of disease was 6.3 years. Table 1 demonstrates the demographics and clinical characteristics of the subjects from all four groups involved in the study. Serum samples were available from 80 subjects at baseline. No statistically significant differences were observed among the four groups in terms of age and gender. Disease severity in AS patients was as follows; 20 subjects had mild disease (BASDAI <4) and 20 patients had moderate or severe disease (BASDAI scores $\geq 4$ ) (Table 2).

The IBD+AS group consisted of 20 subjects including 10 patients with Crohn's disease and 10 UC patients. Of the 10 patients with Crohn's disease, eight had mild disease whereas two had mild-moderate disease according to the CDAI. The Truelove and Witts criteria which was used to assess disease severity in the $10 \mathrm{UC}$ patients revealed only one patient with mild disease, four with moderate disease, and five with severe disease.

In the IBD group which also consisted of 10 Crohn's disease and 10 UC patients, seven out of 10 patients with Crohn's disease had moderate to severe disease, while three were classified as mild to moderate in terms of disease severity.

\begin{tabular}{|c|c|c|c|c|c|c|c|}
\hline \multirow[t]{2}{*}{ Subject groups } & \multirow{2}{*}{$\frac{\text { Number of subjects }}{\mathrm{n}}$} & \multirow{2}{*}{$\frac{\text { Male/Female }}{\mathrm{n}}$} & \multirow{2}{*}{$\frac{\mathrm{UC} / \mathrm{CD}}{\mathrm{n}}$} & \multicolumn{2}{|c|}{ Age (years) } & \multicolumn{2}{|c|}{ Disease duration (years) } \\
\hline & & & & Median & Min.-Max. & Median & Min.-Max. \\
\hline IBD & 20 & $10 / 10$ & $10 / 10$ & 37 & $21-62$ & 2.5 & $1-25$ \\
\hline AS & 20 & $14 / 6$ & $0 / 0$ & 37 & $29-56$ & 7 & $2-20$ \\
\hline IBD+AS & 20 & $11 / 9$ & $10 / 10$ & 40.5 & $19-59$ & 1.5 & $1-13$ \\
\hline Control & 20 & $10 / 10$ & $0 / 0$ & 38.5 & $24-63$ & - & - \\
\hline
\end{tabular}


Table 2. Clinical disease activity in ankylosing spondylitis patients

\begin{tabular}{|c|c|c|c|}
\hline & BASDAI $<4(n=20)$ & BASDAI $\geq 4(n=20)$ & \\
\hline & Median & Median & $p^{*}$ \\
\hline Matrix metalloproteinase-3 (ng/mL) & 6.48 & 40.91 & $<0.05$ \\
\hline Matrix metalloproteinase- 9 ( $\mathrm{ng} / \mathrm{mL})$ & 5.35 & 5.94 & $<0.05$ \\
\hline Tissue inhibitor of matrix metalloproteinase $-1(\mathrm{ng} / \mathrm{mL})$ & 6.16 & 6.61 & $<0.05$ \\
\hline Erythrocyte sedimentation rate $(\mathrm{mm} / \mathrm{hr})$ & 18 & 29 & $<0.05$ \\
\hline C-reactive protein $(\mathrm{mg} / \mathrm{L})$ & 5.5 & 7 & $<0.05$ \\
\hline
\end{tabular}

In addition, eight patients had severe and two had moderate disease out of $10 \mathrm{UC}$ patients.

Human leukocyte antigen B27 was positive in all patients in the AS subgroup. Each of the patients in this subgroup was on anti-tumor necrosis factor (anti-TNF) therapy [infliximab $(n=10)$, adalimumab $(n=8)$, etanercept $(n=2)]$. Eight of the 20 patients in the IBD+AS group were HLA-B27 positive. Also, each patient in this group was on anti-TNF therapy [infliximab $(n=12)$, adalimumab $(\mathrm{n}=8)]$.

Serum MMP-3, MMP-9 and TIMP-1 concentrations, ESR and CRP levels of all subjects are shown in Table 2. Binary comparison showed group differences among MMP-3, MMP-9, and TIMP-1 levels (Table 3).

Significantly higher MMP-3 antigen concentrations $(33.51 \pm 59.56 \mathrm{ng} / \mathrm{mL}, \mathrm{p}=0.045)$ were observed in IBD group when compared to other three groups. In addition, MMP-3 antigen levels were significantly higher in the IBD and IBD+AS group than the AS group alone $(p=0.007$ and $p=0.035$, respectively). MMP-3 antigen levels were also higher in IBD group than IBD+AS and control group alone but the differences were not statistically significant $(p=0.570$ and $p=0.180$, respectively).
The highest MMP-9 antigen levels were seen in the control group $(10.35 \pm 2.61 \mathrm{ng} / \mathrm{mL}, \mathrm{p}=0.048)$. MMP-9 levels in AS group were higher than both IBD and IBD+AS group but the difference was not statistically significant $(p=0.494$ and $p=0.260$, respectively). The higher MMP-9 antigen levels in the control group when compared to patients in the AS or IBD+AS who were on anti-TNF therapy can be explained by the decreasing effect of antiTNF therapy on MMP levels.

Tissue inhibitor of matrix metalloproteinase- 1 antigen levels were highest in the IBD group (8.11 ng/mL, $\mathrm{p}<0.006)$. TIMP-1 levels were also significantly higher in IBD group than both AS and IBD+AS groups $(p=0.033$ and $p=0.008$, respectively).

The correlation between MMM-3, MMP-9, TIMP-1 antigen levels, ESR and CRP values in AS, IBD+AS groups, and BASDAI scores was demonstrated in Table 4. To determine the correlation between biomarkers and disease activity in AS or AS+IBD group, BASDAI scores were calculated independent of treatment. A significant positive correlation was detected between BASDAI scores and MMP-3 antigen levels $(\mathrm{r}=0.841, \mathrm{p}<0.05)$ (Figure 1). In addition, ESR and

\begin{tabular}{|c|c|c|c|c|c|}
\hline Subject groups & $\begin{array}{l}\text { MMP-3 mean } \\
(\mathrm{ng} / \mathrm{mL})\end{array}$ & $\begin{array}{l}\text { MMP-9 mean } \\
\text { (ng/mL) }\end{array}$ & $\begin{array}{l}\text { TIMP-1 mean } \\
(\mathrm{ng} / \mathrm{mL})\end{array}$ & $\begin{array}{l}\text { ESR mean } \\
(\mathrm{mm} / \text { hour })\end{array}$ & $\begin{array}{c}\text { CRP mean } \\
(\mathrm{mg} / \mathrm{L})\end{array}$ \\
\hline IBD & 33.51 & 8.61 & 8.11 & 24.50 & 11.50 \\
\hline AS & 9.13 & 9.37 & 6.61 & 11.50 & 1.00 \\
\hline $\mathrm{IBD}+\mathrm{AS}$ & 20.48 & 3.33 & 5.31 & 30.50 & 13.50 \\
\hline Control & 15.88 & 10.35 & 8.06 & 8.00 & 2.00 \\
\hline$p^{*}$ & 0.045 & 0.048 & 0.006 & 0.029 & 0.004 \\
\hline
\end{tabular}


Table 4. Correlation between biomarkers in ankylosing spondylitis, inflammatory bowel disease + ankylosing spondylitis groups and Bath Ankylosing Spondylitis Disease Activity Index scores

\begin{tabular}{|c|c|c|c|c|c|c|c|}
\hline & $\frac{\text { MMP-3 }}{p}$ & $\frac{\text { MMP-9 }}{p}$ & $\frac{\text { TIMP-1 }}{p}$ & $\frac{\mathrm{ESR}}{p}$ & $\frac{\text { CRP }}{p}$ & $\frac{\text { BASDAI }}{p}$ & $p$ \\
\hline Matrix metalloproteinase-3 & & 0.086 & 0.124 & 0.081 & 0.080 & 0.841 & $<0.05$ \\
\hline Matrix metalloproteinase- 9 & - & - & 0.196 & 0.051 & 0.044 & 0.105 & \\
\hline Tissue inhibitor matrix metalloproteinase -1 & - & - & - & 0.028 & 0.083 & 0.056 & \\
\hline Erythrocyte sedimentation rate & - & - & - & - & 0.889 & 0.231 & $<0.05$ \\
\hline C-reactive protein & - & - & - & - & - & 0.100 & $<0.05$ \\
\hline
\end{tabular}

MMP-3: Matrix metalloproteinase-3; MMP-9: Matrix metalloproteinase-9; TIMP-1: Tissue inhibitor matrix metalloproteinase-1; ESR: Erythrocyte sedimentation rate; CRP: C-reactive protein; BASDAI: Bath Ankylosing Spondylitis Disease Activity Index; Non-parametric Spearman's rank correlation coefficient: $r$ ( $p$ value)

CRP values were not statistically significantly correlated with BASDAI scores $(r=0.231, p>0.05$, and $r=0.100 \mathrm{p}>0.05$ ). There was no correlation between MMP-9 and TIMP-1 antigen levels and BASDAI scores. When patients were classified in terms of mild, moderate and severe disease using the BASDAI scores, significant correlations were detected between MMP-3 antigen levels and disease severity $(p<0.05)$.

Receiver operating characteristic analysis used in comparing the whole spectrum of sensitivity and specificity of three different markers (ESR, CRP and MMP-3) revealed that ESR had 60.4\% sensitivity and $64.5 \%$ specificity; CRP had $59.3 \%$ sensitivity and $61.5 \%$ specificity; MMP-3 had $70.8 \%$ sensitivity and $61.4 \%$ specificity. Non-parametric correlation analysis revealed no significant relation between disease duration and serum MMP-3, MMP-9, and TIMP-1 antigen levels $(p=0.071, p=0.257, p=0.295$, respectively).

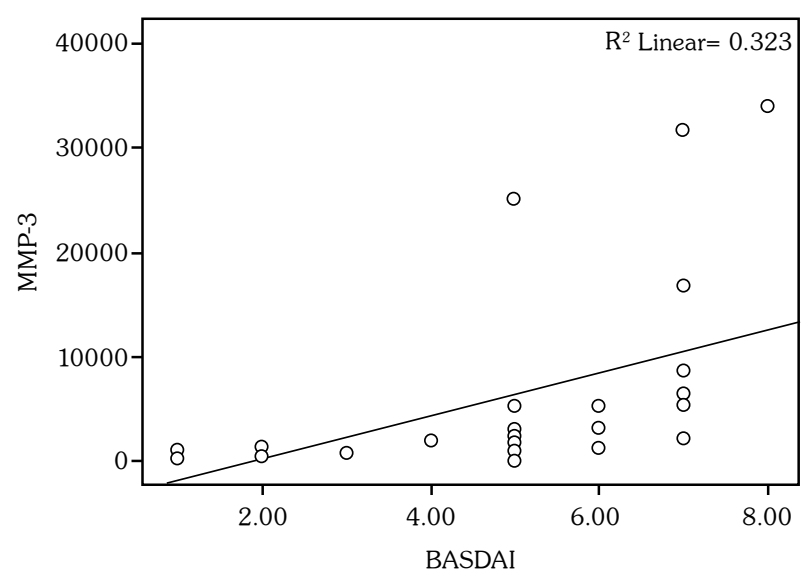

Figure 1. Correlation between MMP-3 and BASDAI. MMP-3: Matrix metalloproteinase-3; BASDAI: Bath Ankylosing Spondylitis Disease Activity Index.

\section{DISCUSSION}

The association of MMPs and TIMP-1 with disease activity and development has barely been investigated in the same experimental setting in SpAs and IBD. While MMP-9 is known to predominantly function in perivascular and intravascular compartments, MMP-3 activation is rather observed in sublining layers. Immunohistochemical studies of synovial tissue samples in SpA patients, conducted by Vandooren et al. ${ }^{21}$ reported expression of MMP-1, MMP-2, MMP-3, MMP-9, TIMP-1 and TIMP-2 on both synovial (lining) and subsynovial (sublining) layers. A statistically positive correlation between MMP expression and inflammatory cellular infiltration, polymorphonuclear cells and vascularity has been shown. ${ }^{20,21}$

Association of disease activity in AS and MMP-3 has already been reported from cohort studies in Beijing, Belgium, and Taiwan. The Beijing cohort showed a significant correlation between MMP-3 and BASDAI scores but revealed no difference when compared with healthy control groups. Likewise, MMP-3 levels were shown to be associated with peripheral involvement in AS according to the Belgian cohort which investigated the role of MMP-3 antigen levels in patients with axial or peripheral involvement. ${ }^{22}$

The largest cohort study was the Taiwan cohort by Chen et al. ${ }^{23}$ The study which evaluated the impact of MMPs and TIMPs on disease activity showed that MMP-3 is a reproducibly sensitive and specific biomarker of disease activity. MMP-3 levels significantly correlated with BASDAI scores and remained stable during a one year follow-up of AS patients. Receiver operating characteristic analysis in this study showed MMP-3 antigen 
levels to be superior than ESR and CRP in determining disease severity with ESR sensitivity of $61.5 \%$ and specificity of $62.5 \%$; CRP sensitivity of $61.5 \%$ and specificity of $62.5 \%$; and MMP-3 sensitivity of $69.2 \%$ and specificity of $68.8 \% .^{23}$

In agreement to previous studies, when we considered possible associations between serum antigen levels of MMP-3, MMP-9, TIMP-1, ESR and CRP, and disease activity in AS, elevated levels of MMP-3 showed significant positive correlations with BASDAI scores in AS and AS+IBD groups. ESR and CRP values were not statistically correlated with BASDAI. These results are consistent with that of the Taiwan cohort. ${ }^{23}$

The effect of TNF-alpha increasing MMP levels in IBD patients with active disease might be the reason for the high MMP-3 levels in IBD group. Also, the vast majority of AS patients on anti-TNF treatment accounts for the low MMP-3 levels in the AS group. Results from Keyszer et al. ${ }^{24}$ study attributing low MMP-3 antigen levels in $\mathrm{SpA}$ patients to absence of cartilage damage in patients with peripheral joint involvement might be another reason for the observes low MMP-3 levels in our study.

We confirm the previous observations made by Lakatos et al. ${ }^{4}$ in their study which demonstrated that serum antigen concentrations TIMP- 1 were significantly increased in patients with UC and $\mathrm{CD}$ compared to controls. These data are in agreement with our study in which TIMP-1 antigen levels were highest in the IBD group $(p<0.006)$.

The most commonly used biomarkers for disease activity in AS are ESR and CRP. Nonetheless, our data did not show correlations between BASDAI and acute-phase reactants (ESR and CRP level). Moreover, although elevated levels of ESR and CRP level were detected in AS patients with high disease activity compared with those with low disease activity, the differences were not statistically significant. This would indicate that, at least in our study, disease activity in AS patients was not well reflected by ESR and CRP level.

In our study, MMP-9 antigen levels were observed to be highest among the control group $(10.35 \pm 2.61 \mathrm{ng} / \mathrm{mL}, \quad p=0.048)$. In addition, although not statistically significant, MMP-9 antigen levels were higher in the AS group compared to both the IBD group and IBD+AS group ( $p=0.494$ and $p=0.260$, respectively). The greater number of AS patients on anti-TNF therapy can be an explanation for this result. In addition, other therapies such as steroids, sulfasalazin, azathioprine, cyclosporine can affect these results in IBD and IBD+AS.

In conclusion, our data confirm the role of MMP-3 as a potential biomarker of disease activity in AS. Together with other MMPs and TIMP-1, these biomarkers appear to be actively involved in the inflammatory processes in AS and IBD. Alongside with other traditional biomarkers such as serum ESR and CRP, serum MMP-3 might be useful additional biomarkers in the assessment of disease activity in AS. The major limitation of this study was the small sample size, and the fact that some levels were significantly low. For this reason, studies with larger sample sizes are required before using MMP-3 in clinical practice. In addition, comparative MMP-3, MMP-9 and TIMP-1 trials are required on diagnosis and post-treatment for evaluating their role on the development and etiopathogenesis of SpAs and IBDs.

\section{Declaration of conflicting interests}

The authors declared no conflicts of interest with respect to the authorship and/or publication of this article.

\section{Funding}

This study was funded by Ankara Tiplılar Vakfı.

\section{REFERENCES}

1. Dougados $M$, van der Linden $S$, Juhlin $R$, Huitfeldt B, Amor B, Calin A, et al. The European Spondylarthropathy Study Group preliminary criteria for the classification of spondylarthropathy. Arthritis Rheum 1991;34:1218-27.

2. Turkcapar N, Toruner M, Soykan I, Aydintug OT, Cetinkaya $\mathrm{H}$, Duzgun $\mathrm{N}$, et al. The prevalence of extraintestinal manifestations and HLA association in patients with inflammatory bowel disease. Rheumatol Int 2006;26:663-8.

3. Chapman RW, Cottone M, Selby WS, Shepherd HA, Sherlock S, Jewell DP. Serum autoantibodies, ulcerative colitis and primary sclerosing cholangitis. Gut 1986;27:86-91.

4. Lakatos G, Hritz I, Varga MZ, Juhász M, Miheller P, Cierny $G$, et al. The impact of matrix metalloproteinases and their tissue inhibitors in inflammatory bowel diseases. Dig Dis 2012;30:289-95. 
5. Hayden DM, Forsyth $\mathrm{C}$, Keshavarzian A. The role of matrix metalloproteinases in intestinal epithelial wound healing during normal and inflammatory states. J Surg Res 2011;168:315-24.

6. Affara NI, Andreu P, Coussens LM. Delineating protease functions during cancer development. Methods Mol Biol 2009;539:1-32.

7. Puthenedam M, Wu F, Shetye A, Michaels A, Rhee KJ, Kwon JH. Matrilysin-1 (MMP7) cleaves galectin-3 and inhibits wound healing in intestinal epithelial cells. Inflamm Bowel Dis 2011;17:260-7.

8. Jensen SA, Vainer B, Bartels A, Brünner N, Sorensen JB. Expression of matrix metalloproteinase 9 (MMP-9) and tissue inhibitor of metalloproteinase 1 (TIMP1) by colorectal cancer cells and adjacent stroma cells-associations with histopathology and patients outcome. Eur J Cancer 2010;46:3233-42.

9. Schrötzlmair F, Kopitz C, Halbgewachs B, Lu F, Algül H, Brünner $\mathrm{N}$, et al. Tissue inhibitor of metalloproteinases1-induced scattered liver metastasis is mediated by host-derived urokinase-type plasminogen activator. $\mathrm{J}$ Cell Mol Med 2010;14:2760-70.

10. Schelter F, Halbgewachs B, Bäumler P, Neu C, Görlach A, Schrötzlmair F, et al. Tissue inhibitor of metalloproteinases-1-induced scattered liver metastasis is mediated by hypoxia-inducible factor- $1 \alpha$. Clin Exp Metastasis 2011;28:91-9.

11. Medina C, Radomski MW: Role of matrix metalloproteinases in intestinal inflammation. $\mathrm{J}$ Pharmacol Exp Ther 2006;318:933-8.

12. Wiercinska-Drapalo A, Jaroszewicz J, Flisiak R, Prokopowicz D. Plasma matrix metalloproteinase-1 and tissue inhibitor of metalloproteinase- 1 as biomarkers of ulcerative colitis activity. World $\mathrm{J}$ Gastroenterol 2003;9:2843-5.

13. Zhu J, Yu DT. Matrix metalloproteinase expression in the spondyloarthropathies. Curr Opin Rheumatol 2006; $18: 364-8$

14. Yang C, Gu J, Rihl M, Baeten D, Huang F, Zhao M, et al. Serum levels of matrix metalloproteinase 3 and macrophage colony-stimulating factor 1 correlate with disease activity in ankylosing spondylitis. Arthritis Rheum 2004;51:691-9.

15. Xia B, Crusius J, Meuwissen S, Pena A. Inflammatory bowel disease: definition, epidemiology, etiologic aspects, and immunogenetic studies. World $\mathrm{J}$ Gastroenterol 1998;4:446-58.

16. Hanauer SB, Sandborn W; Practice Parameters Committee of the American College of Gastroenterology. Management of Crohn's disease in adults. Am J Gastroenterol 2001;96:635-43.

17. Truelove SC, Witts LJ. Cortisone in ulcerative colitis; final report on a therapeutic trial. Br Med J 1955;2:1041-8.

18. Hanauer SB. Inflammtory bowel disease: epidemiology,pathogensis and therapeutic opportunities. Inflamm Bowel Dis 2006:12 Suppl 1:S3-9.

19. Visse R, Nagase H. Matrix metalloproteinases and tissue inhibitors of metalloproteinases structure, function, and biochemistry. Circ Res 2003;92:827-39.

20. Birkedal-Hansen H, Moore WG, Bodden MK, Windsor LJ, Birkedal-Hansen B, DeCarlo A, et al. Matrix metalloproteinases: a review. Crit Rev Oral Biol Med 1993;4:197-250.

21. Vandooren B, Kruithof E, Yu DT, Rihl M, Gu $\mathrm{J}$, De Rycke $\mathrm{L}$, et al. Involvement of matrix metalloproteinases and their inhibitors in peripheral synovitis and down-regulation by tumor necrosis factor alpha blockade in spondylarthropathy. Arthritis Rheum 2004;50:2942-53.

22. van der Linden S, Valkenburg HA, Cats A. Evaluation of diagnostic criteria for ankylosing spondylitis. A proposal for modification of the New York criteria. Arthritis Rheum 1984;27:361-8.

23. Chen $\mathrm{CH}$, Lin KC, Yu DT, Yang C, Huang F, Chen $\mathrm{HA}$, et al. Serum matrix metalloproteinases and tissue inhibitors of metalloproteinases in ankylosing spondylitis: MMP-3 is a reproducibly sensitive and specific biomarker of disease activity. Rheumatology (Oxford) 2006;45:414-20.

24. Keyszer G, Lambiri I, Nagel R, Keysser C, Keysser $\mathrm{M}$, Gromnica-Ihle E, et al. Circulating levels of matrix metalloproteinases MMP-3 and MMP-1, tissue inhibitor metalloproteinases 1 (TIMP-1), and MMP-1/TIMP-1 complex in rheumatic disease. Correlation with clinical activity of rheumatoid arthritis versus other surrogate markers. J Rheumatol 1999;26:251-8. 